\title{
Convergence Theorems for the Variational Inequality Problems and Split Feasibility Problems in Hilbert Spaces
}

\author{
Panisa Lohawech (D), ${ }^{1}$ Anchalee Kaewcharoen ${ }^{(D)},{ }^{1}$ and Ali Farajzadeh ${ }^{2}{ }^{2}$ \\ ${ }^{1}$ Department of Mathematics, Faculty of Science, Naresuan University, Phitsanulok 65000, Thailand \\ ${ }^{2}$ Department of Mathematics, Faculty of Science, Razi University, Kermanshah 67146, Iran \\ Correspondence should be addressed to Anchalee Kaewcharoen; anchaleeka@nu.ac.th
}

Received 20 March 2021; Revised 8 May 2021; Accepted 17 May 2021; Published 3 June 2021

Academic Editor: Sergejs Solovjovs

Copyright (c) 2021 Panisa Lohawech et al. This is an open access article distributed under the Creative Commons Attribution License, which permits unrestricted use, distribution, and reproduction in any medium, provided the original work is properly cited.

\begin{abstract}
In this paper, we establish an iterative algorithm by combining Yamada's hybrid steepest descent method and Wang's algorithm for finding the common solutions of variational inequality problems and split feasibility problems. The strong convergence of the sequence generated by our suggested iterative algorithm to such a common solution is proved in the setting of Hilbert spaces under some suitable assumptions imposed on the parameters. Moreover, we propose iterative algorithms for finding the common solutions of variational inequality problems and multiple-sets split feasibility problems. Finally, we also give numerical examples for illustrating our algorithms.
\end{abstract}

\section{Introduction}

In 2005, Censor et al. [1] introduced the multiple-sets split feasibility problem (MSSFP), which is formulated as follows:

$$
\text { find } x \in \bigcap_{i=1}^{N} C_{i} \text { such that } A x \in \bigcap_{j=1}^{M} Q_{j} \text {, }
$$

where $C_{i}(i=1,2, \ldots, N)$ and $Q_{j}(j=1,2, \ldots, M)$ are nonempty closed convex subsets of Hilbert spaces $H_{1}$ and $H_{2}$, respectively, and $A: H_{1} \longrightarrow H_{2}$ is a bounded linear mapping. Denote by $\Omega$ the set of solutions of MSSFP (1). Many iterative algorithms have been developed to solve the MSSFP (see [1-3]). Moreover, it arises in many fields in the real world, such as inverse problem of intensity-modulated radiation therapy, image reconstruction, and signal processing (see $[1,4,5]$ and the references therein).

When $N=M=1$, the MSSFP is known as the split feasibility problem (SFP); it was first introduced by Censor and Elfving [5], which is formulated as follows:

$$
\text { find } x \in C \text { such that } A x \in Q \text {. }
$$

Denote by $\Gamma$ the set of solutions of SFP (2).

Assume that the SFP is consistent (i.e., (2) has a solution). It is well known that $x \in C$ solves (2) if and only if it solves the fixed point equation

$$
\begin{aligned}
& x=T x, \\
& T=P_{C}\left(I-\gamma A^{*}\left(I-P_{Q}\right) A\right), \quad x \in C,
\end{aligned}
$$

where $\gamma$ is a positive constant, $A^{*}$ is the adjoint operator of $A$, and $P_{C}$ and $P_{Q}$ are the metric projections of $H_{1}$ and $H_{2}$ onto $C$ and $Q$, respectively (for more details, see [6]).

The variational inequality problem (VIP) was introduced by Stampacchia [7], which is finding a point

$$
x^{*} \in C \text { such that }\left\langle F\left(x^{*}\right), x-x^{*}\right\rangle \geq 0, \quad \text { for all } x \in C,
$$

where $C$ is a nonempty closed convex subset of a Hilbert space $H$ and $F: C \longrightarrow H$ is a mapping. The ideas of the VIP are being applied in many fields including mechanics, nonlinear programming, game theory, and economic equilibrium (see [8-12]). 
In [13], we see that $x \in C$ solves (4) if and only if it solves the fixed point equation

$$
\begin{aligned}
& x=S x, \\
& S=P_{C}(I-\mu F), \quad x \in C .
\end{aligned}
$$

Moreover, it is well known that if $F$ is $k$-Lipschitz continuous and $\eta$-strongly monotone, then VIP (4) has a unique solution (see, e.g., [14]).

Since SFP and VIP include some special cases (see $[15,16])$, indeed, convex linear inverse problem and split equality problem are special cases of SFP, and zero point problem and minimization problem are special cases of VIP. Jung [17] studied the common solution of variational inequality problem and split feasibility problem: find a point

$$
x^{*} \in \Gamma \text { such that }\left\langle F x^{*}, x-x^{*}\right\rangle \geq 0, \quad \text { for all } x \in \Gamma,
$$

where $\Gamma$ is the solution set of SFP (2) and $F: H_{1} \longrightarrow H_{1}$ is an $\eta$-strongly monotone and $k$-Lipschitz continuous mapping. After that, for solving problem (6), Buong [2] considered the following algorithms, which were proposed in $[14,18]$, respectively:

$$
\begin{aligned}
& x_{n+1}=\left(I-t_{n} \mu F\right) T x_{n}, \quad n \geq 0, \\
& x_{n+1}=\alpha_{n} x_{n}+\left(1-\alpha_{n}\right)\left(I-t_{n} \mu F\right) T x_{n}, \quad n \geq 0,
\end{aligned}
$$

where $T=P_{C}\left(I-\gamma A^{*}\left(I-P_{Q}\right) A\right)$, and under the following conditions:

$$
\begin{aligned}
& (\mathrm{C} 1) t_{n} \in(0,1), t_{n} \longrightarrow 0 \text { as } n \longrightarrow \infty \text { and } \sum_{n=1}^{\infty} t_{n}=\infty . \\
& \text { (C2) } 0<\liminf _{n \longrightarrow \infty} \alpha_{n} \leq \limsup _{n \longrightarrow \infty} \alpha_{n}<1 .
\end{aligned}
$$

Moreover, Buong [2] considered the sequence $\left\{x_{n}\right\}$ that is generated by the following algorithm, which is weakly convergent to a solution of MSSFP (1):

$$
x_{n+1}=P_{1}\left(I-\gamma A^{*}\left(I-P_{2}\right) A\right) x_{n},
$$

where $P_{1}=P_{C_{1}}, \ldots, P_{C_{N}}$ and $P_{2}=P_{Q_{1}}, \ldots, P_{Q_{M}}$ or $P_{1}=$ $\sum_{i=1}^{N} \alpha_{i} P_{C_{i}}$ and $P_{2}=\sum_{j=1}^{M} \beta_{j} P_{Q_{j}}$ in which $\alpha_{i}$ and $\beta_{j}$, for $1 \leq i \leq N$ and $1 \leq j \leq M$, are positive real numbers such that $\sum_{i=1}^{N} \alpha_{i}=\sum_{j=1}^{M} \beta_{j}=1$.

Motivated by the aforementioned works, we establish an iterative algorithm by combining algorithms (7) and (8) for finding the solution of problem (6) and prove the strong convergence of the sequence generated by our iterative algorithm to the solution of problem (6) in the setting of Hilbert spaces. Moreover, we propose iterative algorithms for solving the common solutions of variational inequality problems and multiple-sets split feasibility problems. Finally, we also give numerical examples for illustrating our algorithms.

\section{Preliminaries}

In order to solve our results, we now recall the following definitions and preliminary results that will be used in the sequel. Throughout this section, let $C$ be a nonempty closed convex subset of a real Hilbert space $H$ with inner product $\langle\cdot, \cdot\rangle$ and norm $\|\cdot\|$.

Definition 1. A mapping $T: H \longrightarrow H$ is called

(i) $k$-Lipschitz continuous, if $\|T x-T y\| \leq k\|x-y\|$ for all $x, y \in H$, where $k$ is a positive number.

(ii) Nonexpansive, if (i) holds with $k=1$.

(iii) $\eta$-strongly monotone, if $\eta\|x-y\|^{2} \leq\langle T x-T y, x-$ $y>$ for all $x, y \in H$, where $\eta$ is a positive number.

(iv) Firmly nonexpansive, if $\|T x-T y\|^{2} \leq\langle T x-T y$, $x-y>$ for all $x, y \in H$.

(v) $\alpha$-Averaged, if $T=(1-\alpha) I+\alpha N$ for some fixed $\alpha \in(0,1)$ and a nonexpansive mapping $N$.

In [5], we know that the metric projection $P_{C}: H \longrightarrow C$ is firmly nonexpansive and (1/2)-averaged.

We collect some basic properties of averaged mappings in the following results.

Lemma 1 (see [16]). We have

(i) The composite of finitely many averaged mappings is averaged. In particular, if $T_{i}$ is $\alpha_{i}$-averaged, where $\alpha_{i} \in(0,1)$ for $i=1,2$, then the composite $T_{1} T_{2}$ is $\alpha$-averaged, where $\alpha=\alpha_{1}+\alpha_{2}-\alpha_{1} \alpha_{2}$.

(ii) If the mappings $\left\{T_{i}\right\}_{i=1}^{N}$ are averaged and have $a$ common fixed point, then

$$
\operatorname{Fix}\left(T_{1}, T_{2}, \ldots, T_{N}\right)=\bigcap_{i=1}^{N} \operatorname{Fix}\left(T_{i}\right)
$$

Proposition 1 (see [19]). Let $D$ be a nonempty subset of $H$, $m \geq 2$ be an integer, and $\phi ;(0,1)^{m} \longrightarrow(0,1)$ be defined by

$$
\phi\left(\alpha_{1}, \ldots, \alpha_{m}\right)=\frac{1}{1+\left(1 / \sum_{i=1}^{m}\left(\alpha_{i} / 1-\alpha_{i}\right)\right)} .
$$

For every $i \in\{1, \ldots, m\}$, let $\alpha_{i} \in(0,1)$ and $T_{i}: D \longrightarrow D$ be $\alpha_{i}$-averaged. Then, $T=T_{1}, \ldots, T_{m}$ is $\alpha$-averaged, where $\alpha=\phi\left(\alpha_{1}, \ldots, \alpha_{m}\right)$.

The following properties of the nonexpansive mappings are very convenient and helpful to use.

Lemma 2 (see [20]). Assume that $H_{1}$ and $H_{2}$ are Hilbert spaces. Let $A: H_{1} \longrightarrow H_{2}$ be a linear bounded mapping such that $A \neq 0$ and let $\mathrm{T}: \mathrm{H}_{2} \longrightarrow \mathrm{H}_{2}$ be a nonexpansive mapping. Then, for $0 \leq \gamma<1 /\|A\|^{2}, I-\gamma A^{*}(I-T) A$ is $\gamma\|A\|^{2}$-averaged.

Proposition 2 (see [19]). Let $C$ be a nonempty subset of $H$, and let $\left\{T_{i}\right\}_{i \in I}$ be a finite family of nonexpansive mappings from $C$ to $H$. Assume that $\left\{\widetilde{\alpha}_{i}\right\}_{i \in I} \subset(0,1)$ and $\left\{\delta_{i}\right\}_{i \in I} \subset(0,1]$ such that $\sum_{i \in I} \delta_{i}=1$. Suppose that, for every $i \in I, T{ }_{i}$ is $\widetilde{\alpha}_{i}$-averaged; then, $T=\sum_{i \in I} \delta_{i} T_{i}$ is $\alpha$-averaged, where $\alpha=\sum_{i \in I} \delta_{i} \widetilde{\alpha}_{i}$.

The following results play a crucial role in the next section.

Lemma 3 (see [14]). Let $t$ be a real number in $(0,1]$. Let $F: H \longrightarrow H$ be an $\eta$-strongly monotone and $k$-Lipschitz 
continuous mapping. The mapping $I-t \mu F$, for each fixed point $\mu \in\left(0,\left(2 \eta / k^{2}\right)\right)$, is contractive with constant $1-t \tau$, i.e.,

$$
\|(I-t \mu F) x-(I-t \mu F) y\| \leq(1-t \tau)\|x-y\|,
$$

where $\tau=1-\sqrt{1-\mu\left(2 \eta-\mu k^{2}\right)} \in(0,1]$.

Theorem 1 (see [21]). Let $F$ be a $k$-Lipschitz continuous and $\eta$-strongly monotone self-mapping of $H$. Assume that $\left\{T_{i}\right\}_{i=1}^{N}$ is a finite family of nonexpansive mappings from $H$ to $H$ such that $C=\cap_{i=1}^{N} \operatorname{Fix}\left(T_{i}\right) \neq \varnothing$. Then, the sequence $\left\{x_{n}\right\}$ defined by the following algorithm converges strongly to the unique solution $x^{*}$ of the variational inequality (4):

$$
x_{n+1}=\left(1-\beta_{n}^{0}\right) x_{n}+\beta_{n}^{0}\left(I-t_{n} \mu F\right) T_{N}^{n}, T_{N-1}^{n}, \ldots, T_{1}^{n} x_{n}, \quad n \geq 0,
$$

where $\mu \in\left(0,2 \eta / k^{2}\right), T_{i}^{n}:=\left(1-\beta_{n}^{i}\right) I+\beta_{n}^{i} T_{i}$, for $i=1, \ldots$, $N$, and under the following conditions:

(i) $t_{n} \in(0,1), t_{n} \longrightarrow 0$ as $n \longrightarrow \infty$ and $\sum_{n=0}^{\infty} t_{n}=\infty$.

(ii) $\beta_{n}^{i} \in(\alpha, \beta)$, for some $\alpha, \beta \in(0,1)$, and $\left|\beta_{n+1}^{i}-\beta_{n}^{i}\right| \longrightarrow 0$ as $n \longrightarrow \infty,(i=0, \ldots, N)$.

Theorem 2 (see [22]). Let F, C, $\mu,\left\{\beta_{n}^{i}\right\}_{i=1}^{N},\left\{t_{n}\right\}$, and $\left\{T_{i}\right\}_{i=1}^{N}$ be as in Theorem 1. Then, the sequence $\left\{x_{n}\right\}$ defined by the following algorithm:

$$
x_{n+1}=\left(I-t_{n} \mu F\right) T_{N}^{n}, T_{N-1}^{n}, \ldots, T_{1}^{n} x_{n}, \quad n \geq 1,
$$

converges strongly to the unique solution $x^{*}$ of variational inequality (4).

\section{Main Results}

In this section, we consider the following iterative algorithm by combining Yamada's hybrid steepest descent method [14] and Wang's algorithm [18] for solving problem (6):

$$
\left\{\begin{array}{l}
y_{n}=\left(1-\alpha_{n}\right) x_{n}+\alpha_{n}\left(I-t_{n} \mu F\right) T x_{n}, \\
x_{n+1}=\left(I-t_{n} \mu F\right) T y_{n}, \quad \forall n \geq 1,
\end{array}\right.
$$

where $T=P_{C}\left(I-\gamma A^{*}\left(I-P_{Q}\right) A\right)$. If we set $\alpha_{n}=0$ for $n \in \mathbb{N}$, then (15) is reduced to (7) studied by Buong [2]. On the other hand, in the Numerical Example section, we present the example illustrating that the two-step method (15) is more efficient that the one-step method (8) studied by Buong [2] and in terms of the two-step method (15) the generated sequence has the less number of iterations and converges faster than the sequence generated by the one-step method (8).

Throughout our results, unless otherwise stated, we assume that $H_{1}$ and $H_{2}$ are two real Hilbert spaces and $A: H_{1} \longrightarrow H_{2}$ is a linear bounded mapping. Let $F$ be an $\eta$-strongly monotone and $k$-Lipschitz continuous mapping on $H_{1}$ with some positive constants $\eta$ and $k$. Assume that $\mu \in\left(0,2 \eta / k^{2}\right)$ is a fixed number.

Theorem 3. Let $C$ and $Q$ be two closed convex subsets in $H_{1}$ and $H_{2}$, respectively. Then, as $n \longrightarrow \infty$, the sequence $\left\{x_{n}\right\}$ defined by (15), where the sequences $\left\{t_{n}\right\}$ and $\left\{\alpha_{n}\right\}$ satisfy conditions (C1) and (C2), respectively, converges strongly to the solution of (6).

Proof. From Lemma 2, we have that $I-\gamma A^{*}\left(I-P_{Q}\right) A$ is $\gamma\|A\|^{2}$-averaged. Since $T=P_{C}\left(I-\gamma A^{*}\left(I-P_{Q}\right) A\right)$, by Lemma 1 (i), we get that $T$ is $\lambda$-averaged where $\lambda=\left(1+\gamma\|A\|^{2} / 2\right)$. Moreover, we obtain that $z \in \Gamma$ if and only if $z \in \operatorname{Fix}(T)$. It follows from Definition 1 (iv) that $T=(1-\lambda) I+\lambda S$, where $S$ is nonexpansive. Then, iterative algorithm (15) can be rewritten as follows:

$$
x_{n+1}=\left(I-t_{n} \mu F\right) T \widetilde{T} x_{n},
$$

where $\widetilde{T}=\left(1-\alpha_{n}\right) I+\alpha_{n}\left(I-t_{n} \mu F\right) T$ and $T=(1-\lambda) I+\lambda S$. Since $(1-\lambda) I+\lambda S$ and $I-t_{n} \mu F$ are nonexpansive, then $(I-$ $\left.t_{n} \mu F\right) T$ is also nonexpansive. Therefore, the strong convergence of (15) to the element $x^{*}$ in the solution set of (6) follows by Theorem 2 .

In [23], Miao and $\mathrm{Li}$ showed the weak convergence results of the sequence $\left\{x_{n}\right\}$ converging to the element of $\operatorname{Fix}(T)$ where $\left\{x_{n}\right\}$ is generated by the following algorithm:

$$
\left\{\begin{array}{l}
y_{n}=\left(1-\beta_{n}\right) x_{n}+\beta_{n}\left(I-t_{n} \mu F\right) T x_{n}, \\
x_{n+1}=\left(1-\alpha_{n}\right) x_{n}+\alpha_{n}\left(I-t_{n} \mu F\right) T y_{n}, \quad \forall n \geq 1,
\end{array}\right.
$$

which $\left\{t_{n}\right\}$ satisfies condition (C3) $\sum_{n=1}^{\infty} t_{n}<+\infty$. Next, we will show the strong convergence for (17) where $\left\{t_{n}\right\}$ satisfies condition (C1).

Theorem 4. Let $C$ and $Q$ be two closed convex subsets in $H_{1}$ and $H_{2}$, respectively. Then, as $n \longrightarrow \infty$, the sequence $\left\{x_{n}\right\}$ defined by (17), where the sequence $\left\{t_{n}\right\}$ satisfies condition (C1) and $\left\{\beta_{n}\right\}$ and $\left\{\alpha_{n}\right\}$ satisfy condition (C2), converges strongly to the solution of (6).

Proof. In the proof of Theorem 3, one can rewrite iterative algorithm (17) as follows:

$$
x_{n+1}=\left(1-\alpha_{n}\right) x_{n}+\alpha_{n}\left(I-t_{n} \mu F\right) T \widetilde{T} x_{n},
$$

where $\widetilde{T}=\left(1-\beta_{n}\right) I+\beta_{n}\left(I-t_{n} \mu F\right) T$ and $T=(1-\lambda) I+\lambda S$. Since $\left(I-t_{n} \mu F\right) T$ is nonexpansive, then the strong convergence of (17) to the element $x^{*}$ in the solution set of (6) follows by Theorem 1 .

Moreover, we obtain the following results which are solving the common solution of variational inequality problem and multiple-sets split feasibility problem, i.e., find a point

$$
x^{*} \in \Omega \text { such that }\left\langle F x^{*}, x-x^{*}\right\rangle \geq 0, \quad \text { for all } x \in \Omega \text {, }
$$

where $\Omega$ is a solution set of (1), and $F: H_{1} \longrightarrow H_{1}$ is an $\eta$-strongly monotone and $k$-Lipschitz continuous mapping. This problem has been studied in [2].

Theorem 5. Let $\left\{C_{i}\right\}_{i=1}^{N}$ and $\left\{Q_{j}\right\}_{j=1}^{M}$ be two finite families of closed convex subsets in $H_{1}$ and $H_{2}$, respectively. Assume that $\gamma \in\left(0,1 /\|A\|^{2}\right),\left\{t_{n}\right\}$ and $\left\{\alpha_{n}\right\}$ satisfy conditions (C1) and (C2), respectively, and the parameters $\left\{\delta_{n}\right\}$ and $\left\{\zeta_{n}\right\}$ satisfy the following conditions: 
(a) $\delta_{i}>0$ for $1 \leq i \leq N$ such that $\sum_{i=1}^{N} \delta_{i}=1$.

(b) $\zeta_{j}>0$ for $1 \leq j \leq M$ such that $\sum_{i=1}^{N} \zeta_{j}=1$.

Then, as $n \longrightarrow \infty$, the sequence $\left\{x_{n}\right\}$, defined by $\left\{\begin{array}{l}y_{n}=\left(1-\alpha_{n}\right) x_{n}+\alpha_{n}\left(I-t_{n} \mu F\right) P_{1}\left(I-\gamma A\left(I-P_{2}\right) A\right) x_{n}, \\ x_{n+1}=\left(I-t_{n} \mu F\right) P_{1}\left(I-\gamma A\left(I-P_{2}\right) A\right) y_{n}, \quad \forall n \geq 1,\end{array}\right.$

with one of the following cases:

(A1) $P_{1}=P_{C_{1}}, \ldots, P_{C_{N}}$ and $P_{2}=P_{Q_{1}}, \ldots, P_{Q_{M}}$

(A2) $P_{1}=\sum_{i=1}^{N} \delta_{i} P_{C_{i}}$ and $P_{2}=\sum_{j=1}^{M} \zeta_{j} P_{Q_{j}}$

(A3) $P_{1}=P_{C_{1}}, \ldots, P_{C_{N}}$ and $P_{2}=\sum_{j=1}^{M} \zeta_{j} P_{Q_{j}}$

(A4) $P_{1}=\sum_{i=1}^{N} \delta_{i} P_{C_{i}}$ and $P_{2}=P_{Q_{1}}, \ldots, P_{Q_{M}}$,

converges to the element $x^{*}$ in the solution set of (19).

Proof. Let $T=P_{1}\left(I-\gamma A^{*}\left(I-P_{2}\right) A\right)$. We will show that $T$ is averaged.

In the case of (A1), $P_{1}=P_{C_{1}}, \ldots, P_{C_{N}}$ and $P_{2}=P_{Q_{1}}, \ldots, P_{Q_{M}}$. Since $P_{C_{i}}$ is $(1 / 2)$-averaged for all $i=1, \ldots, N$, by Proposition 1 , we get that $P_{1}$ is $\lambda_{1}$-averaged, where $\lambda_{1}=N /(N+1)$. Similarly, we have that $P_{2}$ is also averaged and so $P_{2}$ is nonexpansive. By using Lemma 2, we deduce that $I-\gamma A^{*}\left(I-P_{2}\right) A$ is $\lambda_{2}$-averaged, where
TABle 1: Computational results for Example 1 with different methods.

\begin{tabular}{cccccc}
\hline \multicolumn{2}{c}{ Initial point } & \multicolumn{2}{c}{$10^{-4}$} & \multicolumn{2}{c}{$10^{-6}$} \\
\hline & $\begin{array}{c}\text { Buong } \\
\text { method } \\
\text { New } \\
\text { method }\end{array}$ & 29461 & 0.364595 & 2946204 & 31.362283 \\
& 11784 & 0.241371 & 1178481 & 23.411679 \\
\hline$(1,3)^{T}$ & $\begin{array}{c}\text { Buong } \\
\text { method } \\
\text { New } \\
\text { method }\end{array}$ & 12252 & 0.324808 & 1225336 & 25.570356 \\
\hline
\end{tabular}

$\lambda_{2}=\gamma\|A\|^{2}$. It follows from Lemma 1 (i) that $T$ is $\lambda$-averaged with $\lambda=N /(N+1)+\gamma\|A\|^{2}-(N /(N+1)) \gamma\|A\|^{2}$.

If $P_{1}=\sum_{i=1}^{N} \delta_{i} P_{C_{i}}$ and $P_{2}=\sum_{j=1}^{M} \zeta_{j} P_{Q_{j}}$, then by using Proposition 2 and condition (a), we obtain that $P_{1}$ is (1/2)-averaged. From condition (b) and taking into account that $P_{Q_{j}}$ is nonexpansive, for all $j=1, \ldots, M$, we have that $P_{2}$ is also nonexpansive. It follows from Lemma 2 that $I-$ $\gamma A^{*}\left(I-P_{2}\right) A$ is $\gamma\|A\|^{2}$-averaged. Thus, $T$ is $\lambda$-averaged with $\lambda=\left(1+\gamma\|A\|^{2}\right) / 2$.

Cases (A3) and (A4) are similar. This implies that $T:=(1-\lambda) I+\lambda S$, where $S$ is nonexpansive. Moreover, by Lemma 1 , we get that

$$
\begin{aligned}
\operatorname{Fix}(T) & =\operatorname{Fix}\left(P_{1}\right) \cap \operatorname{Fix}\left(I-\gamma A^{*}\left(I-P_{2}\right) A\right)=\operatorname{Fix}\left(P_{1}\right) \cap A^{-1} \operatorname{Fix}\left(P_{2}\right) \\
& =\bigcap_{i=1}^{N} C_{i} \cap A^{-1}\left(\bigcap_{j=1}^{M} Q_{j}\right)=\Omega .
\end{aligned}
$$

Then, iterative algorithm (20) can be rewritten as follows:

$$
x_{n+1}=\left(I-t_{n} \mu F\right) T \widetilde{T} x_{n},
$$

where $\widetilde{T}=\left(1-\alpha_{n}\right) I+\alpha_{n}\left(I-t_{n} \mu F\right) T$ and $T=(1-\lambda) I+\lambda S$. Since $(1-\lambda) I+\lambda S$ and $I-t_{n} \mu F$ are nonexpansive, then ( $I-$ $\left.t_{n} \mu F\right) T$ is nonexpansive. Thus, the strong convergence of
(20) to the element $x^{*}$ in the solution set of (19) follows by Theorem 2.

Theorem 6. Let $\left\{C_{i}\right\}_{i=1}^{N},\left\{Q_{j}\right\}_{j=1}^{M}, \gamma,\left\{t_{n}\right\},\left\{\delta_{n}\right\}$, and $\left\{\zeta_{n}\right\}$ be as in Theorem 5. Then, as $n \longrightarrow \infty$, the sequence $\left\{x_{n}\right\}$, defined by

$$
\left\{\begin{array}{l}
y_{n}=\left(1-\alpha_{n}\right) x_{n}+\alpha_{n}\left(I-t_{n} \mu F\right) P_{1}\left(I-\gamma A\left(I-P_{2}\right) A\right) x_{n}, \\
x_{n+1}=\left(1-\beta_{n}\right) x_{n}+\beta_{n}\left(I-t_{n} \mu F\right) P_{1}\left(I-\gamma A\left(I-P_{2}\right) A\right) y_{n}, \quad \forall n \geq 1,
\end{array}\right.
$$

with one of the cases (A1)-(A4), converges strongly to an element in the solution set of (19).

Proof. In the proof of Theorem 5, one can rewrite iterative algorithm (23) as follows:

$$
x_{n+1}=\left(1-\alpha_{n}\right) x_{n}+\alpha_{n}\left(I-t_{n} \mu F\right) T \widetilde{T} x_{n},
$$

where $\widetilde{T}=\left(1-\beta_{n}\right) I+\beta_{n}\left(I-t_{n} \mu F\right) T$ and $T=(1-\lambda) I+\lambda S$. Since $\left(I-t_{n} \mu F\right) T$ is nonexpansive, the strong convergence of (23) to the element $x^{*}$ in the solution set of (19) follows by Theorem 1.

\section{Numerical Example}

In this section, we present the numerical example comparing algorithm (8) which is given by Buong [2] and algorithm (15) (new method) to solve the following test problem in [2]: find an element $x^{*} \in \Omega$ such that 


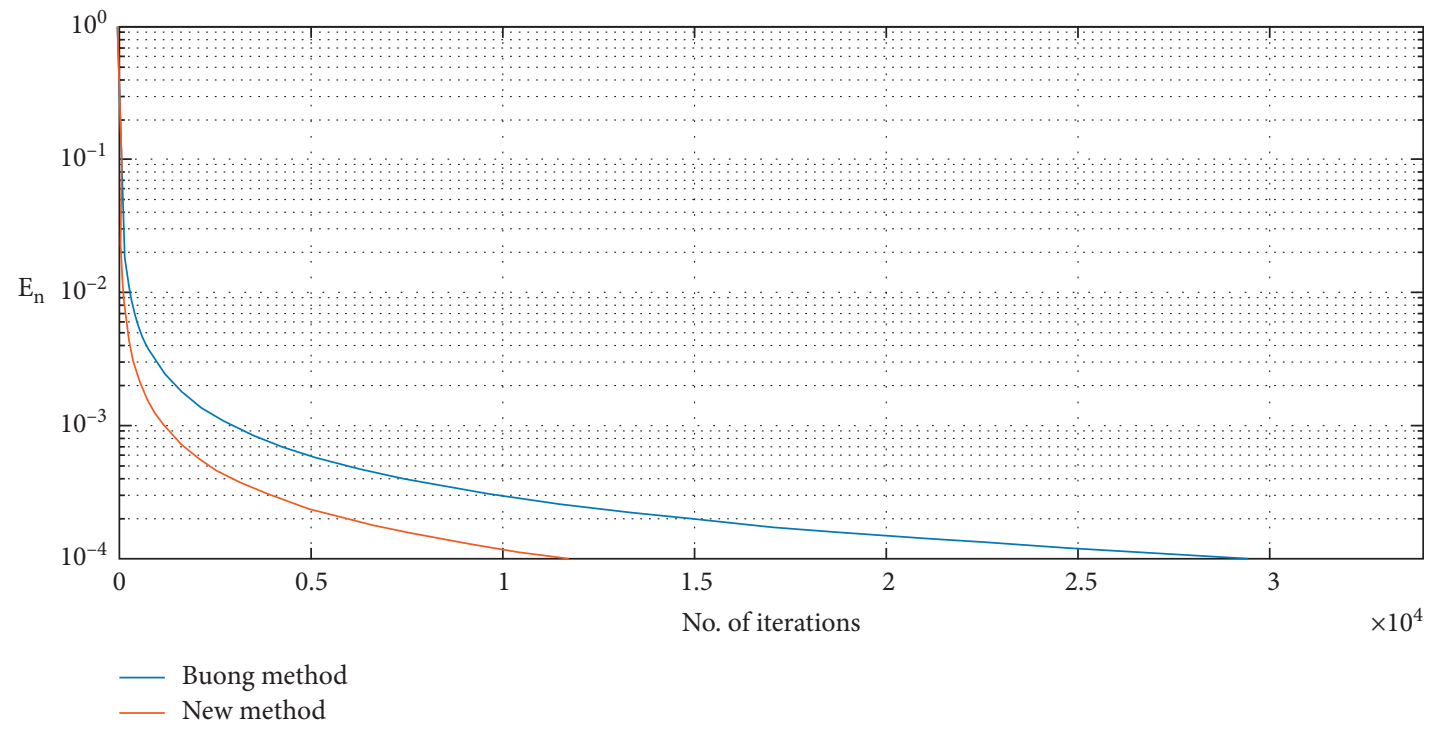

FIgURE 1: The convergence behavior of $E_{n}$ with the initial point $(-2,1)^{T}$.

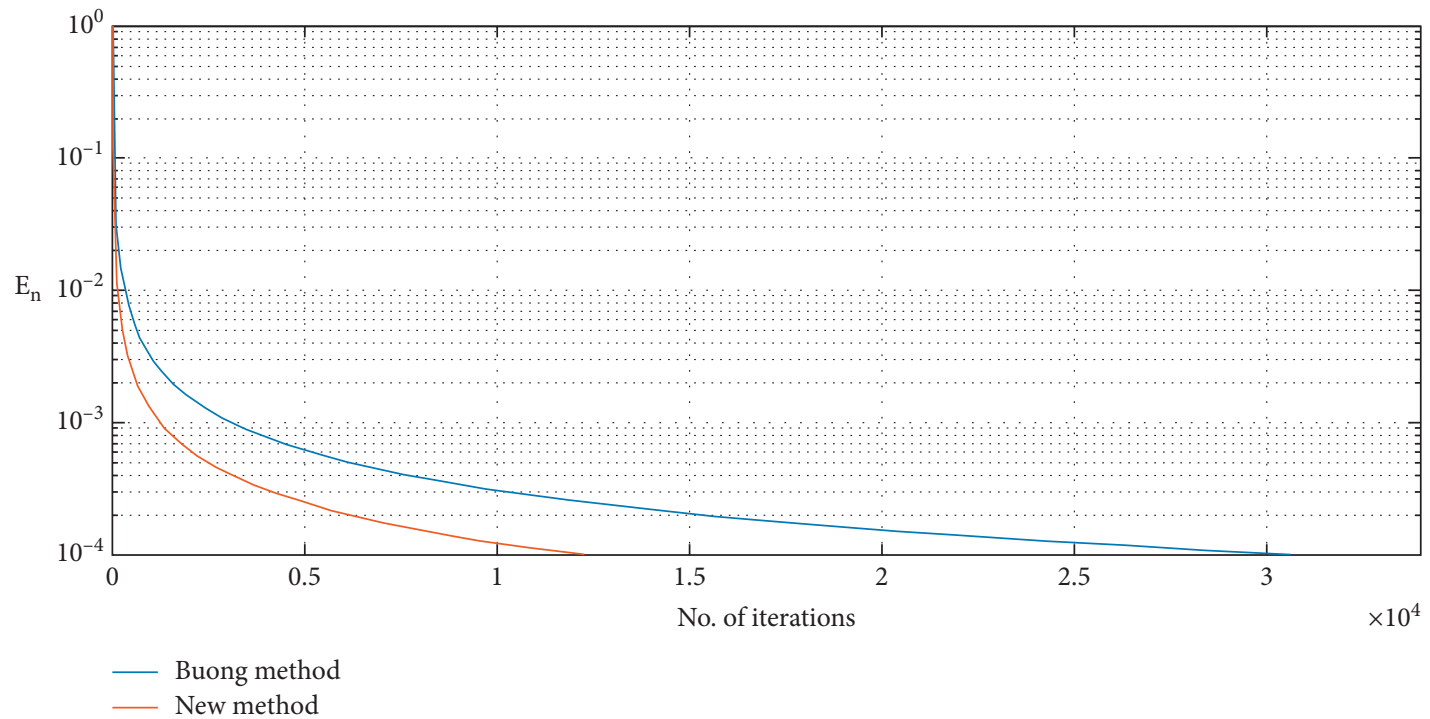

FIgURE 2: The convergence behavior of $E_{n}$ with the initial point $(1,3)^{T}$.

$$
\begin{aligned}
\varphi\left(x^{*}\right) & =\min _{x \in \Omega} \varphi(x), \\
\Omega & =C_{i} \cap A^{-1} Q_{j} \neq \varnothing,
\end{aligned}
$$

where $\varphi$ is a convex function, having a strongly monotone and Lipschitz continuous derivative $\varphi^{\prime}(x)$ on the Euclidian space $\mathbb{E}^{n}, C=\cap_{i=1}^{N} C_{i}$ and $Q=\cap_{j=1}^{M} Q_{j}$ where

$$
C_{i}=\left\{x \in \mathbb{E}^{n}: \sum_{k=1}^{n} a_{k}^{i} x_{k} \leq b_{i}\right\},
$$

$a_{k}^{i}, b_{i} \in(-\infty,+\infty)$, for $1 \leq k \leq n$ and $1 \leq i \leq N$,

$$
Q_{j}=\left\{y \in \mathbb{E}^{m}: \sum_{l=1}^{m}\left(y_{l}-a_{l}^{j}\right)^{2} \leq R_{j}^{2}\right\}, \quad R_{j}>0,
$$

Table 2: Computational results for Example 2 with different methods.

\begin{tabular}{lccccc}
\hline Initial point & & $\mathrm{A} 1$ & $\mathrm{~A} 2$ & $\mathrm{~A} 3$ & $\mathrm{~A} 4$ \\
\hline \multirow{2}{*}{$(-2,1)^{T}$} & $n$ & 28577 & 24264 & 28577 & 24264 \\
& $s$ & 1.491225 & 1.355074 & 1.534414 & 1.282528 \\
\hline \multirow{2}{*}{$(1,3)^{T}$} & $n$ & 33407 & 31438 & 33407 & 31438 \\
& $s$ & 1.746868 & 1.693069 & 1.816897 & 1.690618 \\
\hline
\end{tabular}

$a_{l}^{j} \in(-\infty,+\infty)$, for $1 \leq l \leq m$ and $1 \leq j \leq M$, and $A$ is an $n \times m$-matrix.

Example 1. We consider test problem (25), where $N=M=1, n=m=2, \varphi(x)=(1-a)\|x\|^{2} / 2$ for some fixed $a \in(0,1)$, and 


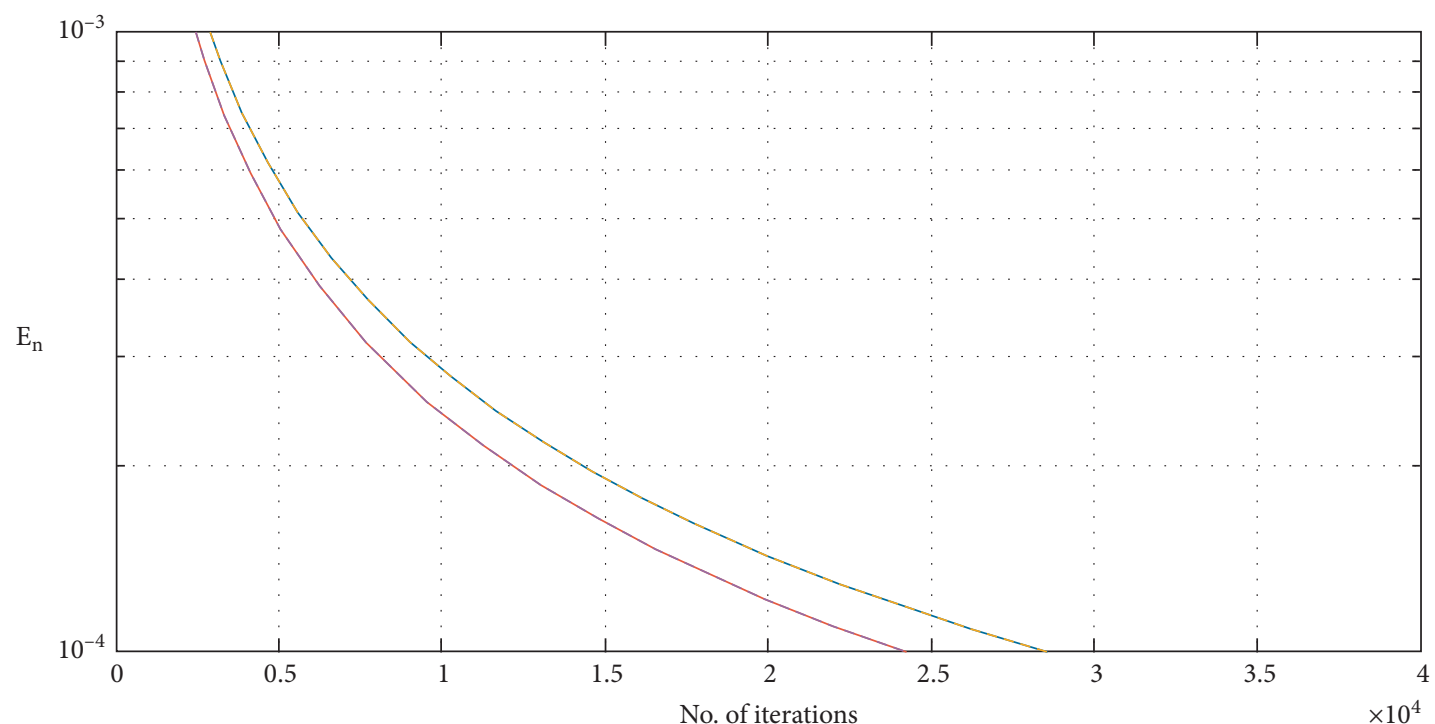
(A1)
(A3)
(A2)
(A4)

Figure 3: The convergence behavior of $E_{n}$ with the initial point $(-2,1)^{T}$.

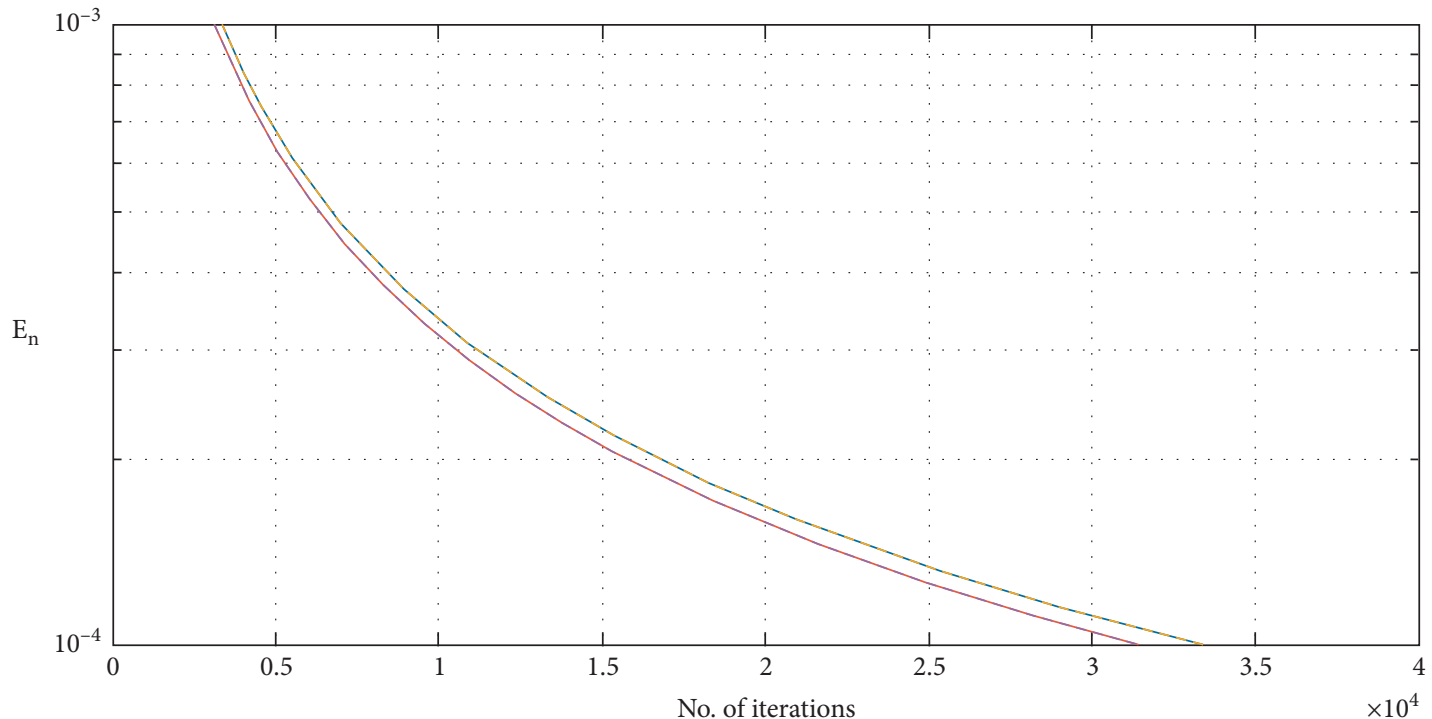

$$
\begin{aligned}
& \text { Figure } 4 \\
& A=\left[\begin{array}{cc}
1 & -1 \\
0 & 2
\end{array}\right] .
\end{aligned}
$$

-.. (A3)

-- (A4)
So, we have that $F$ : $=\varphi^{\prime}=(1-a) I$ is a $k$-Lipschitz continuous and $\eta$-strongly monotone mapping with $k=\eta=(1-a)$. For each algorithm, we set $a^{i}=(1 / i,-1), b_{i}=0$, for all $i=1, \ldots, N$, and $a^{j}=$ $(1 / j, 0), R_{j}=1$, for all $j=1, \ldots, M$. Taking $a=0.5, \gamma=0.3$, the stopping criterion is defined by $E_{n}=\left\|x_{n+1}-x_{n}\right\|<\varepsilon$ where $\varepsilon=10^{-4}$ and $10^{-6}$. The numerical results are listed in Table 1 with different initial points $x^{1}$, where $n$ is the number of
TABLe 3: Computational results for Example 2 with different $\gamma$.

\begin{tabular}{ccccc}
\hline & $\gamma$ & 0.1 & 0.2 & 0.3 \\
\hline \multirow{2}{*}{$(-2,1)^{T}$} & $n$ & 9675 & 19200 & 28577 \\
& $s$ & 0.669508 & 1.245136 & 1.666702 \\
\hline \multirow{2}{*}{$(1,3)^{T}$} & $n$ & 11311 & 22447 & 33407 \\
& $s$ & 0.764536 & 1.372600 & 1.958486 \\
\hline
\end{tabular}

iterations and $s$ is the CPU time in seconds. In Figures 1 and 2, we present the graphs illustrating the number of iterations for both methods using the stopping criterion defined as above with the different initial points shown in Table 1. 
Remark 1. From the numerical analysis of our results in Table 1 and Figures 1 and 2, we get that algorithm (15) (new method) has less number of iterations and faster convergence than algorithm (8) (Buong method).

Example 2. In this example, we consider algorithm (23) for solving test problem (25), where $N=5$ and $M=4$. Let $\left\{C_{i}\right\}_{i=1}^{N},\left\{Q_{j}\right\}_{j=1}^{M}, \varphi, a$, and $A$ be as in Example 1. In the numerical experiment, we take the stopping criterion $E_{n}<10^{-4}$. The numerical results are listed in Table 2 with different cases of $P_{1}$ and $P_{2}$. In Figures 3 and 4 , we present the graphs illustrating the number of iterations for all cases of $P_{1}$ and $P_{2}$ using the stopping criterion as above with the different initial points appeared in Table 2. Moreover, Table 3 shows the effect of different choices of $\gamma$.

Remark 2. We observe from the numerical analysis of Table 2 that algorithm (23) has the fastest convergence when $P_{1}$ and $P_{2}$ satisfy (A4) and the slowest convergence when $P_{1}$ and $P_{2}$ satisfy (A3). Moreover, we require less iteration steps and CPU times for convergence when $\gamma$ is chosen very small and close to zero.

\section{Data Availability}

No data were used to support this study.

\section{Conflicts of Interest}

The authors declare that there are no conflicts of interest.

\section{Acknowledgments}

The first author is thankful to the Science Achievement Scholarship of Thailand. The authors would like to thank the Department of Mathematics, Faculty of Science, Naresuan University (grant no. R2564E049), for the support.

\section{References}

[1] Y. Censor, T. Elfving, N. Kopf, and T. Bortfeld, "The multiplesets split feasibility problem and its applications for inverse problems," Inverse Problems, vol. 21, no. 6, pp. 2071-2084, 2005.

[2] N. Buong, "Iterative algorithms for the multiple-sets split feasibility problem in Hilbert spaces," Numerical Algorithms, vol. 76, no. 3, pp. 783-798, 2017.

[3] J. Zhao, D. Hou, and H. Zong, "Several iterative algorithms for solving the multiple-set split common fixed-point problem of averaged operators," Journal of Nonlinear Functional Analysis, vol. 2019, Article ID 39, 2019.

[4] C. Byrne, "Iterative oblique projection onto convex sets and the split feasibility problem," Inverse Problems, vol. 18, no. 2, pp. 441-453, 2002.

[5] Y. Censor and T. Elfving, "A multiprojection algorithm using bregman projections in a product space," Numerical Algorithms, vol. 8, no. 2, pp. 221-239, 1994.

[6] H. K. Xu, "Iterative methods for the split feasibility problem in infinite-dimensional hilbert spaces," Inverse Problems, vol. 26, no. 10, Article ID 105018, 2010.
[7] G. Stampacchia, "Formes bilineaires coercivites sur les ensembles convexes," Comptes Rendus de l'Académie des Sciences Paris, vol. 258, pp. 4413-4416, 1964.

[8] L. C. Ceng, Q. H. Ansari, and J. C. Yao, "Mann-type steepestdescent and modified hybrid steepest-descent methods for variational inequalities in banach spaces," Numerical Functional Analysis and Optimization, vol. 29, no. 9-10, pp. 9871033, 2008.

[9] L. C. Ceng, M. Teboulle, and J. C. Yao, "Weak convergence of an iterative method for pseudomonotone variational inequalities and fixed-point problems," Journal of Optimization Theory and Applications, vol. 146, no. 1, pp. 19-31, 2010.

[10] M. Fukushima, "A relaxed projection method for variational inequalities," Mathematical Programming, vol. 35, no. 1, pp. 58-70, 1986.

[11] D. Kinderlehrer and G. Stampacchia, An Introduction to Variational Inequalities and their Applications, SIAM, Philadelphia, PA, USA, 2000.

[12] H. Yang and M. G. H. Bell, "Traffic restraint, road pricing and network equilibrium," Transportation Research Part B: Methodological, vol. 31, no. 4, pp. 303-314, 1997.

[13] A. Cegielski, Iterative Methods for Fixed Point Problems in Hilbert Spaces, Springer, Berlin, Germany, 2012.

[14] I. Yamada, "The hybrid steepest descent method for the variational inequality problem over the intersection of fixed point sets of nonexpansive mappings," in Inherently Parallel Algorithms In Feasibility and Optimization and their Applications, D. Butnariu, Y. Censor, and S. Reich, Eds., New York, NY, USA, 2001.

[15] Y. Luo, "An inertial splitting algorithm for solving inclusion problems and its applications to compressed sensing," Journal of Applied and Numerical Optimization.vol. 2, pp. 279-295, 2020.

[16] H. K. Xu, "Averaged mappings and the gradient-projection algorithm," Journal of Optimization Theory and Applications, vol. 150, no. 2, pp. 360-378, 2011.

[17] J. S. Jung, "Iterative algorithms based on the hybrid steepest descent method for the split feasibility problem," Journal of Nonlinear Sciences and Applications, vol. 9, no. 6, pp. 42144225, 2016.

[18] L. Wang, "An iterative method for nonexpansive mapping in hilbert spaces," Journal of Fixed Point Theory and Applications.vol. 2007, p. 8, Article ID 28619, 2007.

[19] P. L. Combettes and I. Yamada, "Compositions and convex combinations of averaged nonexpansive operators," Journal of Mathematical Analysis and Applications, vol. 425, no. 1, pp. 55-70, 2015.

[20] W. Takahashi, H. K. Xu, and J. C. Yao, "Iterative methods for generalized split feasibility problems in Hilbert spaces," SetValued and Variational Analysis, vol. 23, no. 2, pp. 205-221, 2015.

[21] N. Buong and L. T. Duong, "An explicit iterative algorithm for a class of variational iequalities," Journal of Optimization Theory and Applications, vol. 151, no. 3, pp. 513-524, 2011.

[22] H. Zhou and P. Wang, "A simpler explicit iterative algorithm for a class of variational inequalities in hilbert spaces," Journal of Optimization Theory and Applications, vol. 161, no. 3, pp. 716-727, 2014.

[23] Y. Miao and L. Junfen, "Weak and strong convergence of an iterative method for nonexpansive mappings in hilbert spaces," Applicable Analysis and Discrete Mathematics, vol. 2, no. 2, pp. 197-204, 2008. 